\title{
Melanin-Independent Accumulation of Turgor Pressure in Appressoria of Phakopsora pachyrhizi
}

\author{
Hao-Xun Chang, Lou Ann Miller, and Glen L. Hartman
}

First and third authors: Department of Crop Sciences, University of Illinois, Urbana 61801; second author:, Frederick Seitz Material Research Laboratory, University of Illinois, Urbana; and third author: United States Department of Agriculture-Agricultural Research Services, Urbana, IL 61801.

Accepted for publication 16 February 2014.

\begin{abstract}
Chang, H.-X., Miller, L. A., and Hartman, G. L. 2014. Melaninindependent accumulation of turgor pressure in appressoria of Phakopsora pachyrhizi. Phytopathology 104:977-984.

Appressoria of some plant-pathogenic fungi accumulate turgor pressure that produces a mechanical force enabling the direct penetration of hyphae through the epidermis. Melanin functions as an impermeable barrier to osmolytes, which allows appressoria to accumulate high turgor pressure. Deficiency of melanin in appressoria reduces turgor pressure and compromises the infection process. In Phakopsora pachyrhizi, the soybean rust pathogen, the appressoria are hyaline. Our objective was to

ensure the absence of a melanin layer specifically between the appressorial cell wall and plasma membrane, as well as to determine the turgor pressure of $P$. pachyrhizi appressoria. We demonstrated that two melanin biosynthesis inhibitors neither reduced turgor pressure nor compromised the infection process. Transmission electron microscopy also showed the absence of a melanin layer between the appressorial cell wall and plasma membrane. In addition, the turgor pressure of $P$. pachyrhizi appressoria was 5 to $6 \mathrm{MPa}$, based on extracellular osmolytes used to simulate different osmotic pressures. This is the first report showing that turgor pressure accumulation of $P$. pachyrhizi appressoria was independent of melanin.
\end{abstract}

Soybean rust, caused by the fungus Phakopsora pachyrhizi Syd. \& P. Syd., is an economically important disease of soybean that results in significant yield losses $(24,28)$. The infection and colonization of $P$. pachyrhizi in soybean leaves is well documented $(10,18,22,37)$ and reviewed $(13,14)$. Briefly, after a urediniospore is deposited on a leaf, it germinates to form a germ tube that results in a terminal appressorium. The infection continues by the formation of an appressorial cone inside the appressorium, where the penetration hyphae breaks through the plant epidermis, and primary hyphae grow to invade the mesophyll tissue. The hyphae further differentiate into a haustorial mother cell and develop haustoria inside the mesophyll cells. Observation through transmission electron microscopy (TEM) not only elucidated the infection process but also demonstrated that the leaf penetration was dependent on both mechanical force from appressoria and plant cell wall degradation (10).

In many plant-pathogenic fungi, penetration of the plant epidermis occurs by mechanical force as a result of melanized appressoria accumulating high turgor pressure $(2,23)$. Different types of melanin, including dihydroxynaphthalene (DHN) melanin and L-3,4-dihydroxyphenylalanine (DOPA) melanin, are synthesized by different fungi (5). The infection of rice by Magnaporthe oryzae demonstrated the importance of melanized appressoria by treating the fungus with tricyclazole, which inhibits DHN melanin biosynthesis, or by using albino appressorial mutant strains, both of which resulted in limited infection of rice $(7,15,17)$. It was shown that $M$. oryzae accumulated glycerol as an osmolyte inside

Corresponding author: G. L. Hartman; E-mail address: ghartman@illinois.edu

* The $\boldsymbol{e}$-Xtra logo stands for "electronic extra" and indicates that Figures 1, 2, and 3 appear in color online.

http://dx.doi.org/10.1094/PHYTO-12-13-0335-R

This article is in the public domain and not copyrightable. It may be freely reprinted with customary crediting of the source. The American Phytopathological Society, 2014. appressoria that allowed inflow of surrounding water $(9,16)$ and the melanin layer prevented the outflow of osmolytes, resulting in high turgor pressure inside appressoria (27). A similar study has not been done with $P$. pachyrhizi and it is not known whether or not turgor pressure accumulation in appressoria is dependent on melanin. Although the $P$. pachyrhizi appressoria appear to be hyaline, the presence of a melanin layer specifically between the appressorial cell wall and plasma membrane may provide appressoria turgor pressure similar to that demonstrated in $M$. oryzae $(7,15,17)$

Several methods have been developed to measure turgor pressure of appressoria (2). Among these, extracellular osmolytes facilitate the observation of plasmolysis or cytorrhysis of appressoria. For example, appressoria treated with different concentrations of polyethylene glycol (PEG) showed that $8 \mathrm{MPa}$ caused cytorrhysis in $50 \%$ of $M$. oryzae appressoria (16). The same approach also was applied to other plant-pathogenic fungi, including Colletotrichum spp. that also were shown to rely on melanized appressoria for successful infection (23). For Colletotrichum kahawae, the causal agent of coffee berry anthracnose, the DHN melanin synthesis in appressoria allowed for the accumulation of $2.6 \mathrm{MPa}$ turgor pressure, which was shown to be necessary for infection of both leaves and fruit (6). For C. graminicola, which causes anthracnose of maize and other grains, the turgor pressure of appressorium was estimated at 5.4 MPa (3). For Erysiphe graminis f. sp. hordei, the powdery mildew pathogen of barley, a turgor pressure of $2.6 \mathrm{MPa}$ was needed with the assistance of cell-wall-degrading enzyme for penetration and infection (30). Appressorial turgor pressures in plant pathogens have been shown to range from 0.3 to $8 \mathrm{MPa}$ (Table 1 ).

Previous reports indicate that increased turgor pressure may be dependent on melanin accumulation in appressoria. The observation of hyaline appressoria of $P$. pachyrhizi raised questions of whether melanin was absent in the appressorial cell wall or whether a thin melanin layer existed in a way that was invisible to light microscopy, and whether appressoria accumulated turgor 
pressure as reported in other plant fungal pathogens. In this study, the melanized appressoria of soybean anthracnose pathogen, C. truncatum, were used as a comparison to show the differences of turgor pressure accumulation, morphology, and pathogenicity in response to melanin inhibitors. The objective of this study was to determine whether appressoria of $P$. pachyrhizi accumulated turgor pressure and to determine whether the turgor pressure accumulation was independent of melanin synthesis between the appressorial cell wall and the cytoplasm membrane.

\section{MATERIALS AND METHODS}

Fungal and plant materials. The susceptible soybean (Glycine max) 'Williams 82 ' was grown at $25^{\circ} \mathrm{C}$ in a growth chamber set for $12 \mathrm{~h}$ of light $\left(380 \mu \mathrm{mol} \mathrm{m} \mathrm{m}^{-2} \mathrm{~s}^{-1}\right)$ per day. The obligate soybean rust pathogen, $P$. pachyrhizi isolate FL 07-1, was maintained on the first trifoliate detached soybean leaflets. To prepare leaves for inoculation, leaflets were detached from soybean plants and rinsed in $0.1 \%$ Tween 20 (Fisher Scientific, Pittsburgh, PA). Leaflets were placed abaxial side up in clamshell boxes on top of moistened paper towels. Leaflets were inoculated from a previously prepared spore solution $\left(0.65 \times 10^{5}\right.$ spores $\left./ \mathrm{ml}\right)$ until the leaflet was fully covered as the drops dispersed on the Tween 20 rinsed leaflets. The inoculated leaflets were incubated in the dark at $23 \pm 2{ }^{\circ} \mathrm{C}$ for $12 \mathrm{~h}$ and then at $20^{\circ} \mathrm{C}$ with $16 \mathrm{~h}$ of light $(380 \mu \mathrm{mol}$ $\mathrm{m}^{-2} \mathrm{~s}^{-1}$ ) per day. Soybean anthracnose pathogen $C$. truncatum isolate IL28C was maintained on potato dextrose agar (PDA) in a growth chamber set at $25^{\circ} \mathrm{C}$ with $12 \mathrm{~h}$ of light.

The effect of melanin inhibitors on spore germination, appressorial formation, and pathogenicity. The urediniospores of $P$. pachyrhizi and conidia of $C$. truncatum were collected from infected detached soybean leaflets and a culture grown on PDA, respectively. Spores were suspended in $0.1 \%$ Tween 20 solution and adjusted to $0.5 \times 10^{5}$ spores $/ \mathrm{ml}$. Tricyclazole (Sigma-Aldrich, St. Louis) and niacin (Sigma-Aldrich), known melanin inhibitors $(15,20)$, were prepared as $500 \mu \mathrm{M}$ stocks and stored at $4^{\circ} \mathrm{C}$. Tricyclazole was first dissolved in $0.01 \%$ ethanol. Each inhibitor was added to each spore suspension in $0.1 \%$ Tween solution and adjusted to testing concentrations. A $30-\mu$ l spore suspension was spotted on cover glass (No. 1; Corning Inc.) and incubated at $23 \pm$ $2^{\circ} \mathrm{C}$ in the dark. At $6,12,24$, and $48 \mathrm{~h}$ postinoculation (hpi) for $P$. pachyrhizi and 16, 24, and 48 hpi for C. truncatum, spore germination and appressoria formation (with or without melanin inhibitors) were measured under the Olympus compound microscope BX51. This was conducted three times using 50 spores for each of the three biological replications, totaling 450 spore observations for each fungus at each time point.

To determine the effect of the melanin inhibitors on pathogenicity of the two fungi, spores collected as previously described were suspended in $50 \mu \mathrm{M}$ niacin, $50 \mu \mathrm{M}$ tricyclazole, and $0.1 \%$ Tween 20 at a concentration of $0.65 \times 10^{5}$ and $3 \times 10^{6}$ spores $/ \mathrm{ml}$ for $P$. pachyrhizi and $C$. truncatum, respectively. The detached leaflets were uniformly sprayed with each spore suspension and incubated at $23 \pm 2{ }^{\circ} \mathrm{C}$ in the dark for 12 and $36 \mathrm{~h}$ for $P$. pachyrhizi and $C$. truncatum, respectively. The leaflets were arranged in moist chambers in a completely random design, with three leaflets per treatment. The leaflets were incubated for 10 days at $20^{\circ} \mathrm{C}$ with $16 \mathrm{~h}$ of light for P. pachyrhizi and at $25^{\circ} \mathrm{C}$ with $12 \mathrm{~h}$ of light for $C$. truncatum. To quantify $P$. pachyrhizi uredinia, two 5-mm leaf disks were randomly sampled per leaflet from three randomly selected leaflets. The leaf disks were bleached in a mixture of absolute ethanol-acetic acid (3:1 [ $\mathrm{vol} / \mathrm{vol}])$ for $12 \mathrm{~h}$ to remove pigments $(4,37)$. The leaf disks were then floated in lactophenol (Fisher Scientific) for $6 \mathrm{~h}$ and stained with $0.1 \%$ cotton blue (ICN Pharmaceuticals Inc.) in lactophenol for $12 \mathrm{~h}$. The leaf disks were washed with distilled water twice and stained uredinia were examined visually under the Olympus stereoscope SZX16. Three biological replications were conducted.

Turgor pressure measurements and permeability differences between the cell walls of urediniospores and appressoria. PEG 8000 (Sigma-Aldrich) solutions were used to conduct plasmolysis tests. The conversion of PEG concentration to osmotic pressure $\Pi(\mathrm{MPa})$ is given by the equation $\Pi=\alpha C+\beta C^{2}$, where $\Pi$ is the osmotic pressure (MPa), $C$ is the molar concentration (M) of PEG, and $\alpha$ and $\beta$ are constants from different molecular weights of PEGs (26). The $\alpha$ and $\beta$ constant were interpolated based on the regression equation for $\alpha$ as $y \alpha=(-2 \times$ $\left.10^{-7}\right) x^{2}-0.0021 x+1.4356, R^{2}=0.9487$; and $\beta$ as $y \beta=(4 \times$ $\left.10^{-5}\right) x^{2}-0.0547 x+41.444, R^{2}=0.9969$; with the $y$ as the speculated constant from the regression equation and $x$ the molecular weight of PEGs (16). Based on the analysis, the $\alpha$ and $\beta$ constants for PEG 8000 were -28.16 and 2,163.84, respectively. Hence, the solutions in different osmotic pressures were prepared based on the transfer equation $\Pi=-28.16 C+2,163.84 C^{2}$. A 3.22 molar glycerol (Fisher Scientific) was prepared to generate the $5.8 \mathrm{MPa}$ osmotic pressure, as previously reported (9).

For the turgor pressure measurements, the water drop on the cover glass was removed with a Kimwipe (Kimberly-Clark, Knoxville, TN) and replaced with $30 \mu \mathrm{l}$ of PEG 8000 solution at 6, 12, 24, and 48 hpi for P. pachyrhizi and 16, 24, and 48 hpi for C. truncatum. After 5 min of incubation with PEG 8000 solution, the number of appressoria with plasmolysis was counted. For each of the osmotic PEG 8000 solution treatments, 100 spores were counted at each time point with three biological replications. Linear regression analysis was used to determine the concentration that caused $50 \%$ plasmolysis of the appressoria and was used as the turgor pressure of the appressoria as previously described in other fungal appressoria $(6,16)$.

To quantify the permeability of the cell wall of urediniospores, a 5.8-MPa glycerol solution together with a 6-MPa PEG 8000 solution as applied to appressoria $12 \mathrm{hpi}$ for $5 \mathrm{~min}$. In this experiment, there were 100 spores counted within each of three biological replications.

Data analyses. The data collected from replicated experiments were subjected to analysis of variance using R 3.0.1 (R Foundation for Statistical Computing, Vienna). If the linear model was significant, Tukey's honestly significant difference test (R 3.0.1) was used to separate means. The variables analyzed included the

TABLE 1. Documented appressorial turgor pressures in different fungal plant pathogens ${ }^{\mathrm{a}}$

\begin{tabular}{|c|c|c|c|c|c|}
\hline Phylum & Species & Pressure (MPa) & Melanin & Method & Reference \\
\hline \multirow[t]{2}{*}{ Ascomycota } & Magnaporthe oryzae & 8 & Present & PEG 8000 cytorrhysis & 16 \\
\hline & M. oryzae & 5.8 & Present & Glycerol cytorrhysis & 9 \\
\hline Ascomycota & Colletotrichum kahawae & 2.6 & Present & PEG 8000 plasmolysis & 6 \\
\hline \multirow[t]{2}{*}{ Ascomycota } & C. graminicola & 5.35 & Present & Waveguide deformation & 3 \\
\hline & C. graminicola & 3.9 & Present & Mach-Zehnder-Microscopy and cytorrhysis & 21 \\
\hline Ascomycota & Erysiphe graminis f. sp. hordei & $2-4$ & ND & PEG6000 plasmolysis & 30 \\
\hline Ascomycota & C. truncatum & $4-5$ & Present & PEG 8000 plasmolysis & This study \\
\hline Basidiomycota & Uromyces spp. & $0.27-0.35$ & ND & ND & 30,33 \\
\hline Basidiomycota & Phakopsora pachyrhizi & $5-6$ & Absent & PEG 8000 plasmolysis & This study \\
\hline
\end{tabular}

a $\mathrm{PEG}=$ polyethylene glycol and $\mathrm{ND}=$ not described. 
number of uredinia stained in plant tissues to determine the effect of melanin inhibitors on pathogenicity, turgor pressure measurements of appressoria in the experiments that used tricyclazole and niacin, and the percentage of plasmolysis and cytorrhysis that resulted when urediniospores were treated with PEG and glycerol.

TEM observations. Soybean leaflets containing appressoria 12 hpi were embedded in $2 \%$ agar. The leaf tissues were fixed in Karnovsky's Fixative in phosphate-buffered 2\% glutaraldeyde and $2.5 \%$ paraformaldehyde. The tissue was washed in Sorenson's phosphate buffer at $\mathrm{pH} 7.2$ with no further additives, and infiltrated by $2 \%$ osmium tetroxide for $2 \mathrm{~h}$ followed by the addition of $3 \%$ potassium ferrocyanide for $30 \mathrm{~min}$. After rinsing with water, saturated uranyl acetate was added for en bloc staining. A vacuum was used to facilitate the efficiency of infiltrations using a vacuum jar connected to a pump (Air Cedet Portable Vacuum/ Pressure Station; Barnant Thermo Scientific, Barrington, IL).

The tissue was dehydrated in a series of increasing concentrations of ethanol. Acetonitrile was used as the transition fluid between ethanol and the epoxy. An infiltration series was done with an epoxy mixture using the epon substitute Lx112. The resulting blocks were polymerized at $80^{\circ} \mathrm{C}$ for $12 \mathrm{~h}$, trimmed, and sectioned with diamond knives. Sections were stained with filtered uranyl acetate and Luft's instant lead citrate (Ladd Research Inc., Williston, VT) following previously described procedures $(12,25)$, then examined and photographed with a Hitachi H600 Transmission Electron Microscope.

\section{RESULTS}

The effect of melanin inhibitors on spore germination, appressorial formation, and pathogenicity. To avoid obser- vation bias under the light microscope and to support the independence of melanin synthesis during appressoria formation of $P$. pachyrhizi, we applied DHN and DOPA melanin inhibitors tricyclazole and niacin, respectively, to ensure the absence of melanin in P. pachyrhizi appressoria. There was no effect of the melanin inhibitors on spore germination and appressorial formation of $P$. pachyrhizi and $C$. truncatum spores. The appressoria of $P$. pachyrhizi were hyaline, with no pigmentation on the appressorial cell wall. In contrast, the appressoria of $C$. truncatum were opaque and melanized. Appressoria of $P$. pachyrhizi treated with tricyclazole and niacin were visually identical to the appressoria in the $0.1 \%$ Tween 20 solution control. Appressoria of C. truncatum treated with $50 \mu \mathrm{M}$ tricyclazole blocked melanin synthesis and turned the intermediate product into reddish compounds $(7,11,36)$, whereas niacin did not eliminate the melanin, indicating that the opaque color was DHN melanin (Fig. 1).

Soybean leaflets inoculated with conidia of $C$. truncatum mixed with tricyclazole produced fewer necrotic spots than conidia mixed in niacin or in the Tween 20 control (Fig. 2A). Soybean leaflets inoculated with urediniospores of $P$. pachyrhizi suspended in tricyclazole or niacin were visually indistinguishable from the control based on the appearance of sporulating uredinia (Fig. 2B). Based on counts of stained uredinia (Fig. 2C), there were no significant differences between the treatments (Fig. 2D).

Turgor pressure measurements and permeability differences between the cell walls of urediniospores and appressoria. A fully mature appressorium of $P$. pachyrhizi was defined as one in which all the cytoplasm had migrated and was delimited from the germ tube into the appressorium. The highest appressorium turgor pressure was $6.01 \pm 0.93 \mathrm{MPa}$ at $12 \mathrm{hpi}$. The accumulation peaked at $12 \mathrm{hpi}$ and decreased till 48 hpi. Neither

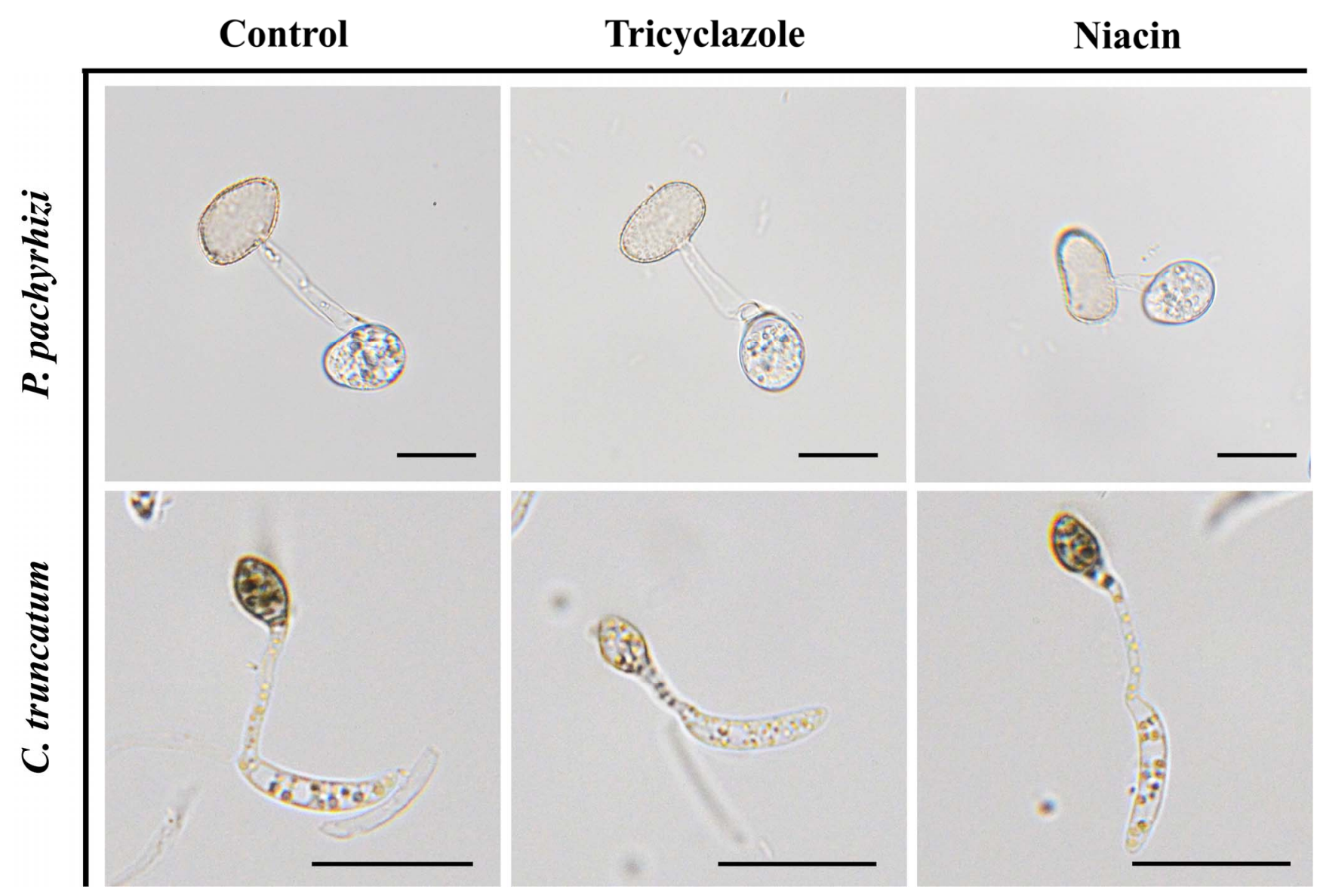

Fig. 1. Nonmelanized appressoria of Phakopsora pachyrhizi. Germination of urediniospores of P. pachyrhizi and conidia of Colletotrichum truncatum, and the formation of appressoria. Both melanin inhibitors had no impact on P. pachyrhizi appressoria. In contrast, the melanized appressorium of $C$. truncatum became light reddish and more transparent in the presence of dihydroxynaphthalene melanin inhibitor tricyclazole. Bar represents $25 \mu \mathrm{m}$ for all pictures. 

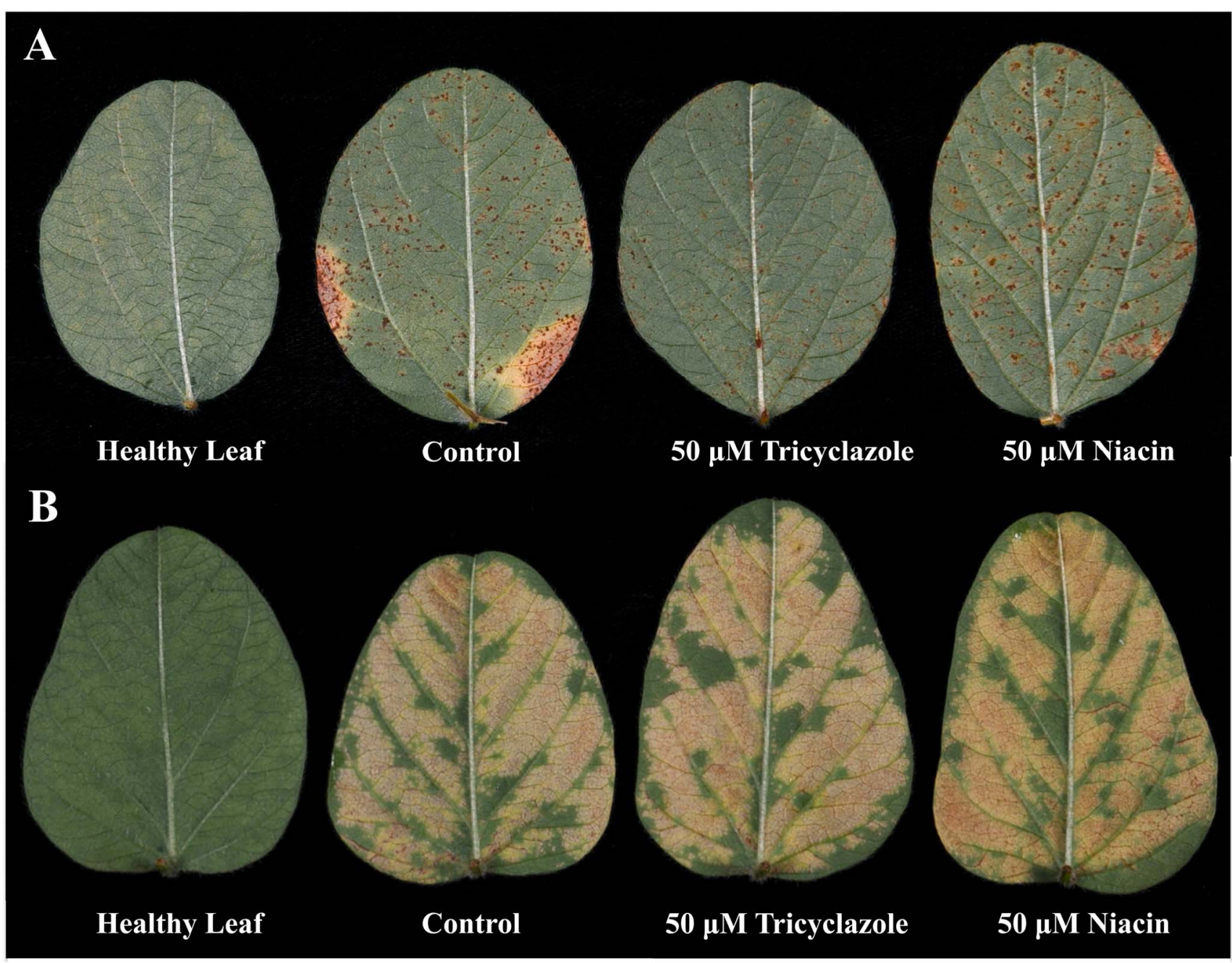

C

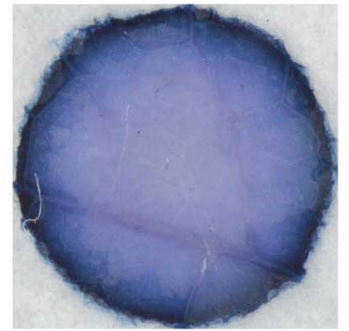

Healthy Leaf

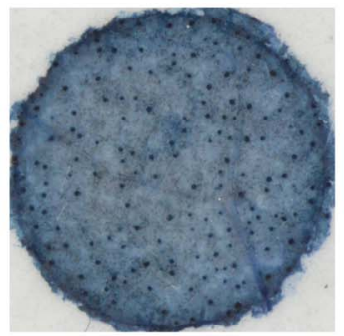

$50 \mu \mathrm{M}$ Tricyclazole

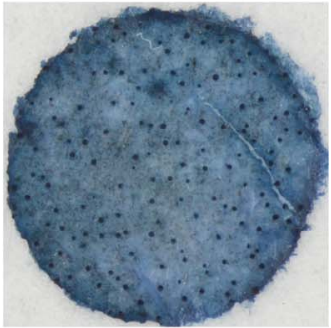

Control

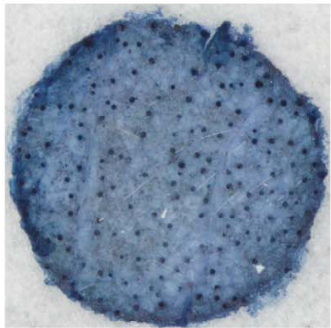

$50 \mu \mathrm{M}$ Niacin
D

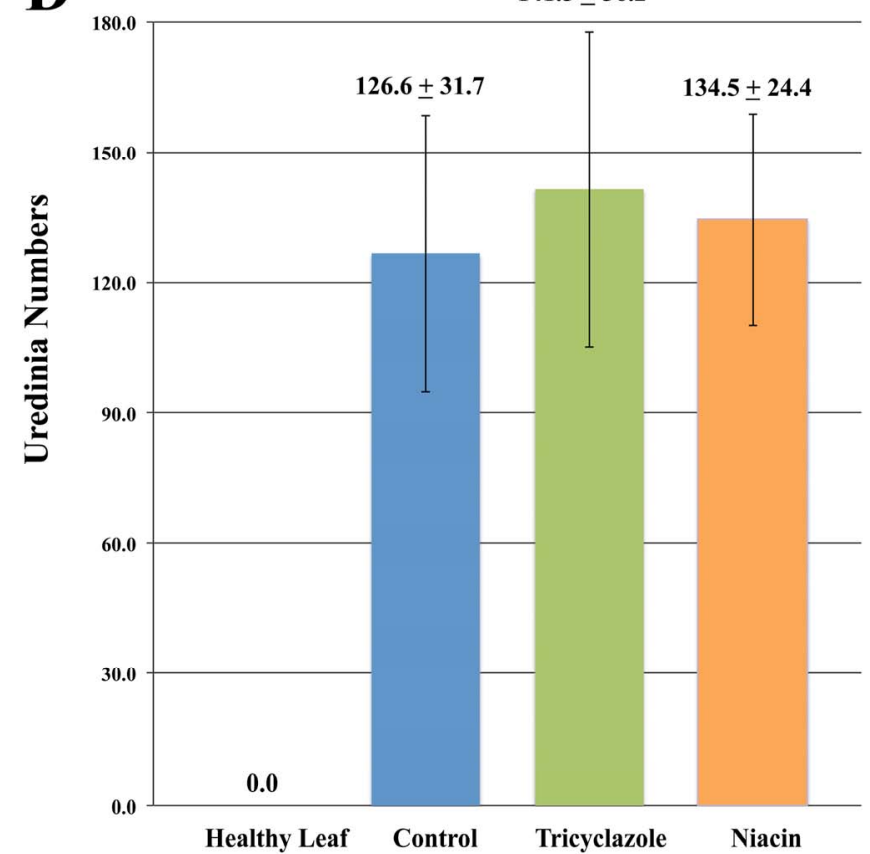

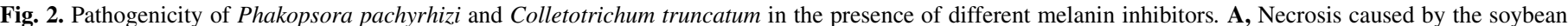

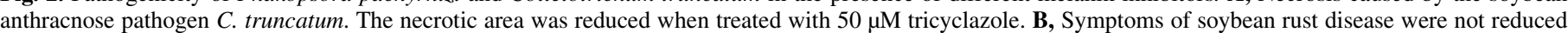

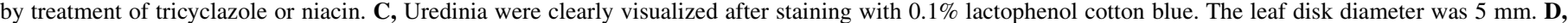

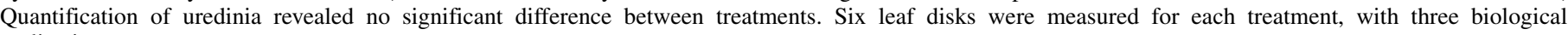
replications. 
tricyclazole nor niacin affected the accumulation of turgor pressure (Table 2). When the melanin inhibitors were used at different concentrations $(50,150$, and $250 \mu \mathrm{M})$, there was no significant reduction in turgor pressure of appressoria of $P$. pachyrhizi (Table 3).

A fully mature appressorium of $C$. truncatum was defined as an appressorium completely melanized, or approximately the same size of the swollen tip of the germination tube when tricyclazole was applied. The highest appressorium turgor pressure was $4.58 \pm$ $0.25 \mathrm{MPa}$ at $24 \mathrm{hpi}$. Niacin did not alter turgor pressure accumulation; however, tricyclazole significantly $(P<0.05)$ reduced the pressure at $16 \mathrm{hpi}(3.56 \pm 0.29 \mathrm{MPa})$ and $24 \mathrm{hpi}(2.79 \pm$ $0.15 \mathrm{MPa}$ ) (Table 2).

Based on the treatments of 6-MPa PEG 8000 and 5.8-MPa glycerol solutions, plasmolysis of $P$. pachyrhizi appressoria was observed. In both treatments, the cell wall remained at the original position while the cytosol shrank inward and left a space inside the appressorium (Fig. 3). The empty urediniospores responded to PEG 8000 and glycerol differently. Only $10.67 \pm 2.52 \%$ of empty urediniospores had cytorrhysis, while the majority remained unchanged under glycerol osmotic pressure. However, the $73.67 \pm$ $10.02 \%$ of empty urediniospores exhibited cytorrhysis under the osmotic pressure generated by the PEG 8000 solution, which showed a significantly different response to the molecular size $(P<0.05)$. These observations suggested that both the large molecule PEG 8000 and the small molecule glycerol were permeable through the nonmelanized cell wall of $P$. pachyrhizi appressoria but the permeability was different in the case of the empty urediniospores.

TEM observations. The appressoria had lost their spherical shape upon penetration. In response to infection, soybean cells would form papillae to counteract the infection at the penetration site (Fig. 4A). When penetration began, an appressorial cone was observed (Fig. 4A and $\mathrm{C}$ to $\mathrm{E}$ ). The penetration hyphae invaded through the epidermis and developed into primary hyphae, which colonized the leaf tissue (Fig. 4E). However, no melanin layer was observed in the cell walls of appressoria in all these stages, although a gray waxy layer was found locally (Fig. 4B to D). Although dark material was associated with the appressorial cone near the penetration site, the accumulation of this dark material

TABLE 3. Appressorial turgor pressures (MPa) of Phakopsora pachyrhizi in different concentrations of melanin inhibitors at $12 \mathrm{~h}$ postinoculation

\begin{tabular}{lll}
\hline Concentration $(\mu \mathrm{M})^{\mathrm{a}}$ & Tricyclazole & Niacin \\
\hline 0 & & $4.90 \pm 0.24$ \\
50 & $4.91 \pm 0.43$ & $4.88 \pm 0.23$ \\
150 & $4.70 \pm 0.24$ & $4.53 \pm 0.17$ \\
250 & $4.70 \pm 0.28$ & $4.66 \pm 0.19$ \\
\hline
\end{tabular}

a Spores were suspended in $0.1 \%$ Tween 20 aqueous solution as a control condition. Tricyclazole and niacin were also suspended in $0.1 \%$ Tween 20 aqueous solution. In all, 150 spores were counted for each osmolality conditions, with a total of 1,200 spores measured to construct a regression line. Each value represents three biological replications.

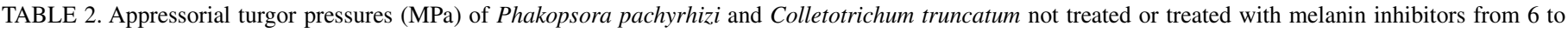
$48 \mathrm{~h}$ postinoculation (hpi)

\begin{tabular}{|c|c|c|c|c|c|c|c|}
\hline \multirow[b]{2}{*}{ Treatment $^{\mathrm{a}}$} & \multicolumn{4}{|c|}{ P. pachyrhizi } & \multicolumn{3}{|c|}{ C. truncatum } \\
\hline & $6 \mathrm{hpi}$ & 12 hpi & $24 \mathrm{hpi}$ & 48 hpi & $16 \mathrm{hpi}$ & 24 hpi & 48 hpi \\
\hline Control & $3.95 \pm 0.38$ & $6.05 \pm 0.73$ & $4.26 \pm 0.32$ & $2.73 \pm 0.22$ & $4.18 \pm 0.05$ & $4.58 \pm 0.25$ & $3.18 \pm 0.40$ \\
\hline Tricyclazole & $4.05 \pm 0.25$ & $6.01 \pm 0.93$ & $4.23 \pm 0.29$ & $2.11 \pm 0.54$ & $3.56 \pm 0.29^{b}$ & $2.79 \pm 0.15^{\mathrm{b}}$ & $2.87 \pm 0.39$ \\
\hline Niacin & $4.14 \pm 0.26$ & $5.87 \pm 0.56$ & $4.13 \pm 0.50$ & $1.83 \pm 0.42$ & $4.29 \pm 0.33$ & $4.38 \pm 0.22$ & $3.95 \pm 0.59$ \\
\hline
\end{tabular}

a Spores were suspended in $0.1 \%$ Tween 20 aqueous solution. Tricyclazole and Niacin were also suspended in $0.1 \%$ Tween 20 aqueous solution. In all, 100 spores were counted for each osmolality conditions for each time point, with a total of 500 spores measured to construct a regression line. Each value represents three biological replications.

b Significantly different from control $(P<0.05)$.
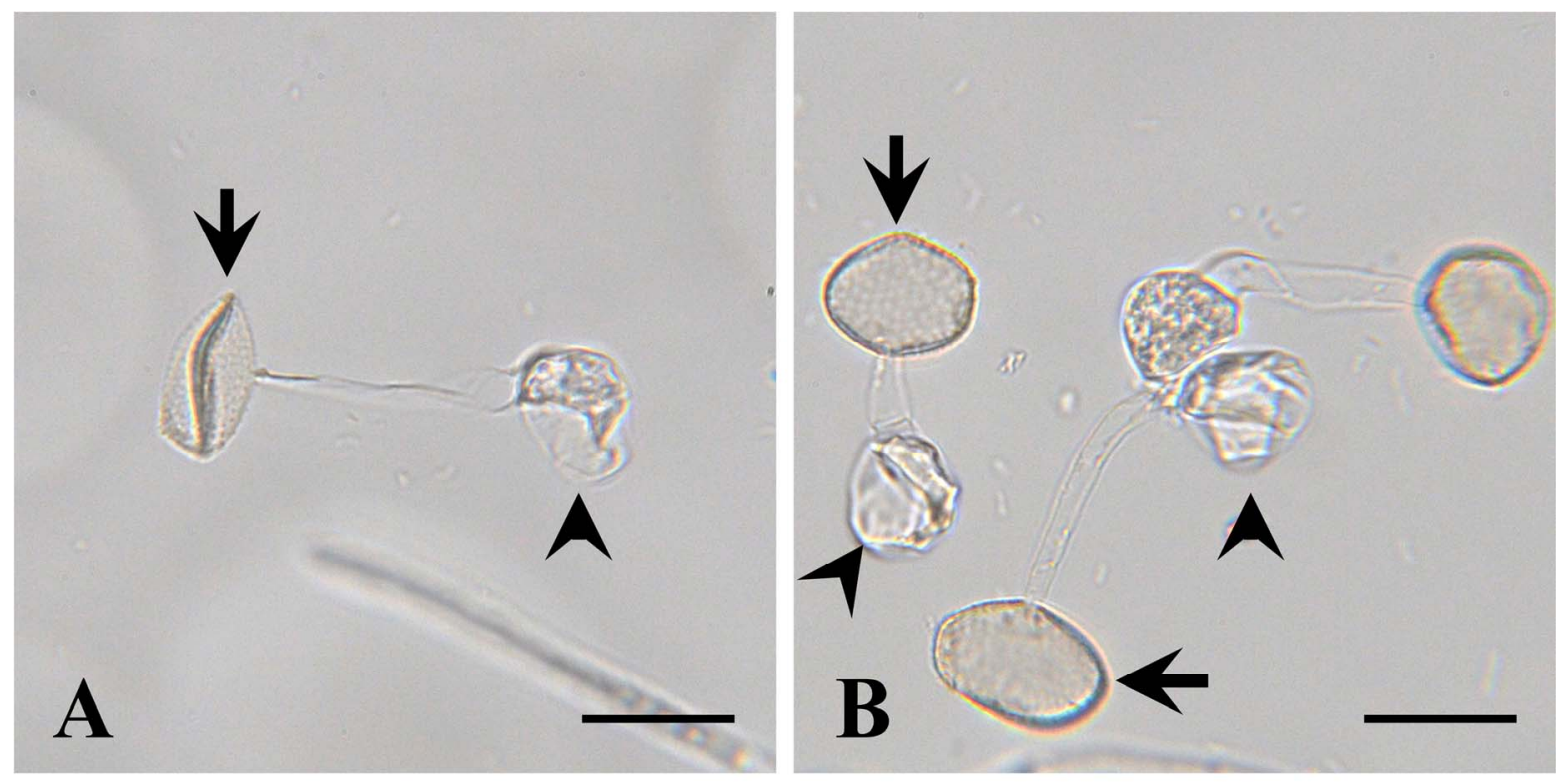

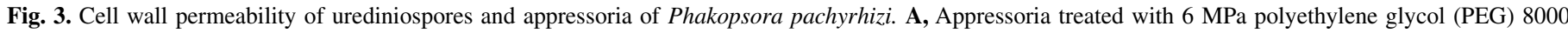

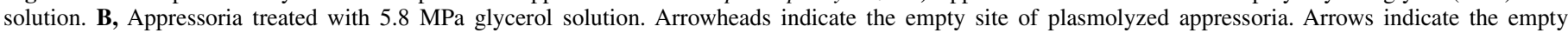

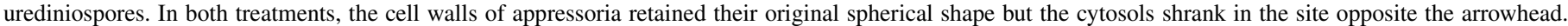

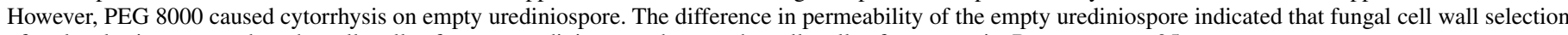
of molecule size occurred on the cell walls of empty urediniospores but not the cell walls of appressoria. Bars represent $25 \mu \mathrm{m}$. 

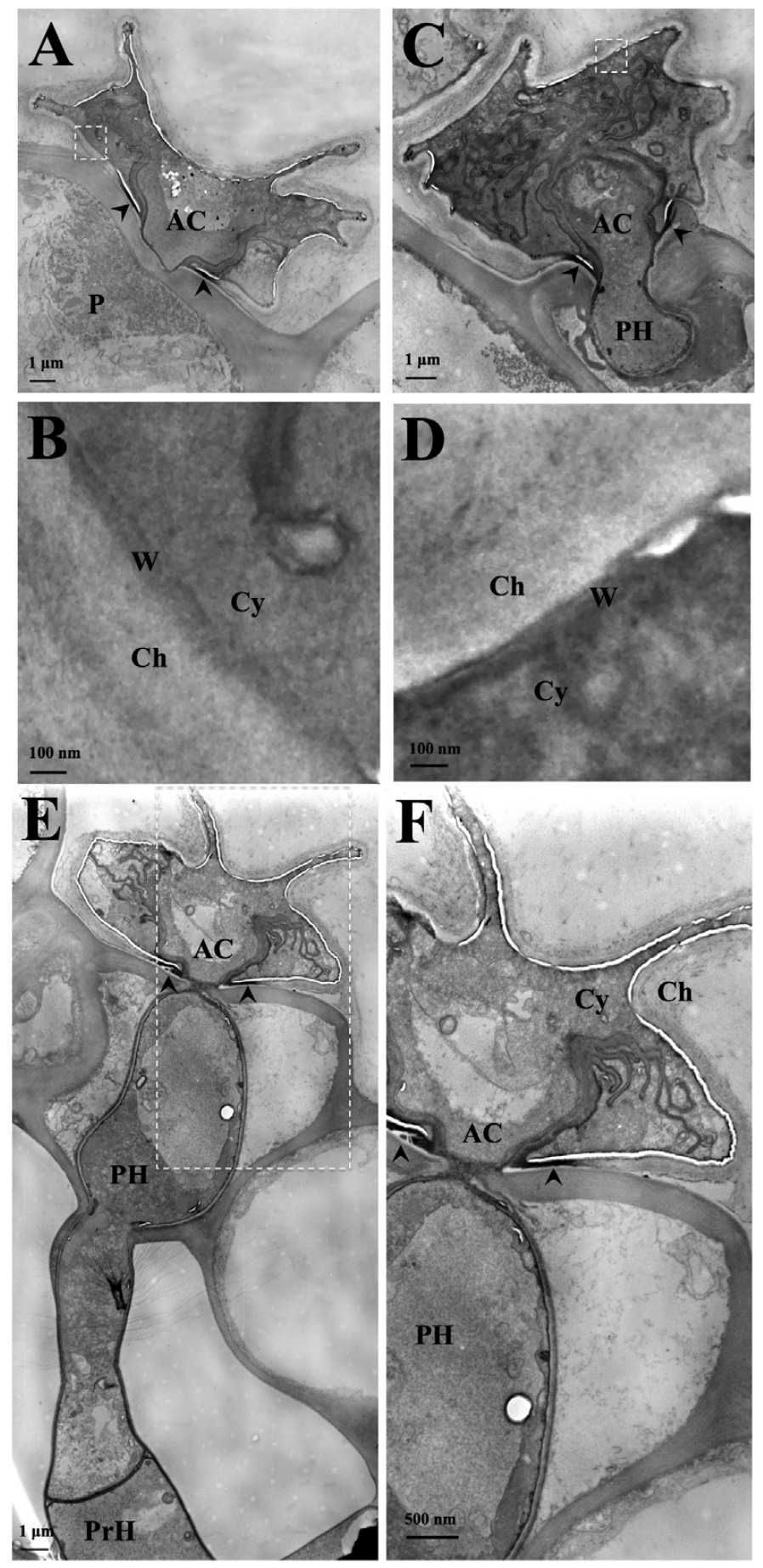

Fig. 4. Transmission electron microscopy analysis of material between appressorial cell walls and cytoplasmic membrane of Phakopsora pachyrhizi. $\mathrm{AC}=$ appressorial cone, $\mathrm{Ch}=$ chitin, $\mathrm{Cy}=$ cytosol, $\mathrm{P}=$ papillae, $\mathrm{PH}=$ penetration hyphae, $\mathrm{PrH}=$ primary hyphae, $\mathrm{W}=$ waxy layer; arrowhead indicates dark materials. A, Early stage of penetration. Papillae formed to prevent the invasion of $P$. pachyrhizi penetration hyphae. Appressorial cone started to form at this stage. B, Amplified image of the white dash block in A. No dark material could be found in between chitin cell wall and cytosol. However, a gray waxy layer presented in between. C, Penetration hyphae developed and invaded into epidermis. Dark material became more obvious only around the penetration site and did not surround the appressorium or was not observed between the appressorial cell wall and cytosol. D, Amplified image of the white dash block in C. Gray waxy layer became more obvious. E, Penetration hyphae invaded through the epidermis, and developed into primary hyphae for further colonization. F, Enlarged image of the white dash block in E. Dark material was limited at penetration site but not other parts between the cell wall and cytosol of the appressorium. did not follow the appressorial cell wall (Fig. 4B and D to F), indicating the absence of a melanin layer between the cytosol and appressorial cell wall of $P$. pachyrhizi.

\section{DISCUSSION}

The accumulation of turgor pressure in appressoria of $P$. pachyrhizi was independent of melanin. The DHN and DOPA melanin inhibitors tricyclazole and niacin did not alter the development or morphology of appressoria, nor was there any interruption of the infection process. In comparison, the turgor pressure of C. truncatum declined significantly from 4.58 to $2.79 \mathrm{MPa}$ and the melanin in appressoria disappeared in the presence of the DHN melanin inhibitor tricyclazole. When treated with hyperosmotic PEG 8000 solution, most of the appressoria of $P$. pachyrhizi plasmolyzed, with the appressorial cell wall remaining in position and the detached cytosol shrinking inward. When treated with hyperosmotic glycerol, nearly all of the appressoria plasmolyzed similarly to the PEG 8000 treatment. However, the empty urediniospores responded differently; the PEG solution caused cytorrhysis in the empty urediniospores and plasmolysis of the appressoria whereas glycerol caused plasmolysis in both empty urediniospores and appressoria. This indicated that the appressorial cell walls of $P$. pachyrhizi were permeable to small molecules of large polymers such as PEG 8000; however, the cell walls of urediniospores were only permeable to small molecules such as glycerol, reported to have the ability to diffuse through the nonmelanized appressorial cell wall in M. oryzae (9).

We did not observe a melanin layer in the TEM micrographs at any of the stages of appressoria formation and penetration. We observed that spherical appressoria collapsed or deformed upon penetration, consistent with a previous report (18), and similar to a report on a closely related rust fungus $P$. apoda, where appressoria were observed as wrinkled or polymorphic rather than spherical at the penetration stage (1). The observed penetration process was consistent with previous reports $(10,18)$ but we noticed an unidentified gray layer or substance existing between the appressorial cell wall and cytosol. The layer is different from dark melanin and likely a waxy layer or a polysaccharide layer that serves to join the appressorial cell wall and plasma membrane. The waxy layer may make a minor contribution to preventing the outflow of cytosol substances because the appressorial cell wall was permeable to molecules as large as PEG 8000 in our tests.

Fungi produce different kinds of melanin that function primarily to protect fungal structures and aid in survival. Based on the composition of their monomer, they are either DOPA melanin or DHN melanin, and both enhance cell tolerance against radiation, extreme redox, temperature conditions, and degradation of enzymes (5). In addition, melanized appressoria allow the accumulation of high turgor pressure, which generates the mechanical force to penetrate plant cell walls $(8,17,23)$.

The identification of the melanin inhibitor tricyclazole and the melanin-deficient mutants in the rice blast fungus $M$. oryzae led to the discovery that melanin was essential for pathogenicity and launched studies that focused on the function of melanin in appressoria $(7,15)$. The location of DHN melanin between the plasma membrane and the appressorial cell wall was first characterized by TEM, and further supported by discovering that the melanin layer disappeared with treatment of tricyclazole. The melanin layer was impermeable to molecules larger than water, based on high concentrations of $\mathrm{KCl}$ solution treatments, which allowed the appressoria to maintain osmolytes inside and recruit water to maintain turgor pressure (15). The first report of measuring the appressorial turgor pressure of $M$. oryzae used extracellular PEG solutions differing in osmolality to estimate the osmotic pressure that equates to $50 \%$ cytorrhysis and found that the turgor pressure in the appressoria was up to $8 \mathrm{MPa}$ (16). The 
osmolytes in the appressoria of $M$. oryzae were characterized as mainly glycerol, which were shown to leak out of appressoria in melanin-deficient mutants (9). Cytorrhysis of appressoria occurred when hyperosmotic glycerol was applied for both wildtypes and melanin-deficient mutants; however, the melanindeficient appressoria recovered through plasmolysis by the equilibrium of extracellular hyperosmotic glycerol (9). With this evidence, the authors concluded that glycerol was capable of diffusing between the melanin-deficient appressorial cell walls but not the melanized appressorial cell walls. Therefore, the function of the melanin layer was shown to prevent the leakage of osmolytes such as glycerol through appressorial cell walls $(9,27)$. An alternative function for melanin in appressoria recently suggested that, instead of a build-up of impermeability, melanin prevents the deleterious damage of cell-wall-degrading enzymes produced by the fungus, and it was shown that melanized and nonmelanized appressoria of $C$. graminicola did not differ from the concentration of osmolytes based on measurements made by using a Mach-Zehnder-Microscopy; however, the nonmelanized appressoria were more sensitive to extracellular lysing enzymes (21). This suggested that the outflow of osmolytes through the plasma membrane could be limited (21). If this is the case, and the lipid bilayer is indeed sufficient for maintaining high osmolytes in the cytosol, the measurements of lower glycerol in melanin-deficient strains of $M$. oryzae compared with the wild type may not be plausible, as was previously reported (9). Because melanin does not appear to be required for turgor pressure accumulation of $P$. pachyrhizi and in the melanin-deficient mutants of $C$. graminicola (21), it is not clear how appressoria maintain osmolytes and generate high turgor pressure.

In $M$. oryzae, DHN melanin was shown to be responsible for the dark appressoria. The pathogenicity was reduced in mutants deficient in the DHN melanin synthesis pathway (7). In the DHN melanin synthesis pathway, polyketide synthase (PKS) polymerizes the precursor acetate that terminates in $1,8-\mathrm{DHN}$ as the monomer for the melanin polymer. For the albino mutants (pks-) of $M$. oryzae, C. lagenarium, and $C$. graminicola, they were not able to produce melanin $(7,21,32)$. Several expressed sequence tag (EST) and RNA-Seq studies completed during urediniospore germination and appressoria or uredinia formation reported no PKS genes in $P$. pachyrhizi $(29,31,34,35)$. Although the sensitivity on detecting PKS genes by EST and RNA-Seq methods depends on the coverage and depth of sample libraries, the absence of PKS gene detection during urediniospore germination and appressoria formation may suggest that the expression of PKS genes is not necessary for $P$. pachyrhizi at these stages. Furthermore, a genome-wide study also indicated that the orthologs of PKS genes rarely exist in fungi belonging to the subdivision Pucciniomycotina, including Melampsora laricis-populina, Mixia osmundae IAM 14324, Puccinia graminis, Rhodotorula graminis strain WP1, and Sporobolomyces roseus (19). As one of the members in Pucciniomycotina, it seems likely that Phakopsora pachyrhizi has no PKS genes. The whole-genome sequencing will provide advanced details on the genome structure of P. pachyrhizi.

This appears to be the first case of melanin-independent turgor pressure accumulation in $P$. pachyrhizi appressoria. Melanin inhibitors failed to affect the appressorial morphology, development, and turgor pressure or the pathogenicity of $P$. pachyrhizi. No melanin layer was observed between the cytosols and appressorial cell walls based on TEM micrographs, even though the appressorial turgor pressure of $P$. pachyrhizi was measured at 5 to $6 \mathrm{MPa}$. Our study provides evidence to support the idea that melanin may not be needed to build up an impermeable barrier, because turgor pressure accumulation was independent of melanin. It remains unclear how $P$. pachyrhizi manages the osmolytes and turgor pressure inside appressoria without creating an impermeable barrier.

\section{ACKNOWLEDGMENTS}

We thank R. Vittal and H.-C. Yang, University of Illinois, for their advice on working with pathogens causing soybean rust and soybean anthracnose; and T. Herman for her review of this manuscript.

\section{LITERATURE CITED}

1. Adendorff, A., and Rijkemberg, F. H. J. 2000. Scanning electron microscopy of direct host leaf penetration by urediospore-derived infection structures of Phakopsora apoda. Mycol. Res. 104:217-324.

2. Bastmeyer, M., Deising, H. B., and Bechinger, C. 2002. Force exertion in fungal infection. Annu. Rev. Biophys. Biomol. Struct. 31:321-341.

3. Bechinger, C., Giebel, K.-F., Schnell, M., Leiderer, P., Deising, H. B., and Bastmeyer, M. 1999. Optical measurements of invasive forces exerted by appressoria of a plant-pathogenic fungus. Science 285:1896-1899.

4. Bonde, M. R., Nester, S. E., Austin, C. N., Stone, C. L., Frederick, R. D., Hartman, G. L., and Miles, M. R. 2006. Evaluation of virulence of Phakopsora pachyrhizi and P. meibomiae isolates. Plant Dis. 90:708-716.

5. Butler, M. J., and Day, A. W. 1998. Fungal melanins: a review. Can. J. Microbiol. 44:1115-1136.

6. Chen, Z., Nunes, M. A., Silva, M. C., and Rodrigues, C. J., Jr. 2004. Appressorium turgor pressure of Colletotrichum kahawae might have a role in coffee cuticle penetration. Mycologia 96:1199-1208.

7. Chumley, F. G., and Valent, B. 1990. Genetic analysis of melanindeficient, nonpathogenic mutants of Magnaporthe grisea. Mol. PlantMicrobe Interact. 3:135-143.

8. Deising, H. B., Werner, S., and Wernitz, M. 2000. The role of fungal appressoria in plant infection. Microbes Infect. 2:1631-1641.

9. de Jong, J. C., McCormack, B. J., Smirnoff, N., and Talbot, N. J. 1997. Glycerol generates turgor in rice blast. Nature 389:244-245.

10. Edwards, H. H., and Bonde, M. R. 2011. Penetration and establishment of Phakopsora pachyrhizi in soybean leaves as observed by transmission electron microscopy. Phytopathology 101:894-900.

11. Engh, I., Nowrousian, M., and Kuck, U. 2007. Regulation of melanin biosynthesis via the dihydroxynaphthalene pathway is dependent on sexual development in the ascomycete Sordaria macrospora. FEMS Microbiol. Lett. 275:62-70.

12. Glauert, A. 1980. Practical Methods in Electron Microscopy. Part 1: Fixation, Dehydration and Embedding of Biological Specimens. Elsevier North-Holland Inc., Amsterdam, Netherlands.

13. Goellner, K., Loehrer, M., Langenbach, C., Conrath, U., Koch, E., and Schaffrath, U. 2010. Phakopsora pachyrhizi, the causal agent of Asian soybean rust. Mol. Plant Pathol. 11:169-177.

14. Hartman, G. L., Hill, C. B., Twizeyimana, M., Miles, M. R., and Bandyopadhyay, R. 2011. Interaction of soybean and Phakopsora pachyrhizi, the cause of soybean rust. CAB Rev. Perspect. Agric. Vet. Sci. Nutr. Nat. Resources 6. doi:10.1079/PAVSNNR20116025

15. Howard, R. J., and Ferrari, M. A. 1989. Role of melanin in appressorium function. Exp. Mycol. 13:403-418.

16. Howard, R. J., Ferrari, M. A., Roach, D. H., and Money, N. P. 1991. Penetration of hard substrates by a fungus employing enormous turgor pressures. Proc. Natl. Acad. Sci. USA 88:11281-11284.

17. Howard, R. J., and Valent, B. 1996. Breaking and entering: host penetration by the fungal rice blast pathogen Magnaporthe grisea. Annu. Rev. Microbiol. 50:491-512.

18. Koch, E., Ebrahim Nesbat, F., and Hoppe, H. H. 1983. Light and electron microscopic studies on the development of soybean rust (Phakopsora pachyrhizi Syd.) in susceptible soybean leaves. Phytopathol. Z. 106:302320 .

19. Lackner, G., Misiek, M., Braesel, J., and Hoffmeister, D. 2012. Genome mining reveals the evolutionary origin and biosynthetic potential of basidiomycete polyketide synthases. Fungal Genet. Biol. 49:996-1003.

20. Lee, J.-K., Jung, H.-M., and Kim, S.-Y. 2003. 1,8-Dihydroxynaphthalene (DHN)-melanin biosynthesis inhibitors increase erythritol production in Torula corallina, and DHN-melanin inhibits erythrose reductase. Appl. Environ. Microbiol. 69:3427-3434.

21. Ludwig, N., Lohrer, M., Hempel, M., Mathea, S., Schliebner, I., Menzel, M., Kiesow, A., Schaffrath, U., Deising, H., and Horbach, R. 2014. Melanin is not required for turgor generation but enhances cell wall rigidity in appressoria of the corn pathogen Colletotrichum graminicola. Mol. Plant-Microbe Interact. 27:315-327.

22. McLean, R. J. 1979. Histological studies of resistance to soybean rust, Phakopsora pachyrhizi Syd. Aust. J. Agric. Res. 30:77-84.

23. Mendgen, K., Hahn, M., and Deising, H. 1996. Morphogenesis and mechanisms of penetration by plant-pathogenic fungi. Annu. Rev. Phytopathol. 34:367-386.

24. Miles, M. R., Levy, C., Morel, W., Mueller, T., Steinlage, T., van Rij, N., Frederick, R. D., and Hartman, G. L. 2007. International fungicide 
efficacy trials for the management of soybean rust. Plant Dis. 91:14501458.

25. Miller, L. 2001. Microwave processing techniques for biological samples in a service laboratory. In: Techniques and Protocols. M. Giberson and R. Demaree, eds. Humana Press Inc., Totowa, NJ.

26. Money, N. P. 1989. Osmotic pressure of aqueous polyethylene glycols: relationship between molecular weight and vapor pressure deficit. Plant Physiol. 91:766-769.

27. Money, N. P. 1997. Mechanism linking cellular pigmentation and pathogenicity in rice blast disease. Fungal Genet. Biol. 22:151-152.

28. Mueller, T. A., Miles, M. R., Morel, W., Marios, J. J., Wright, D. L., Kemerait, R. C., Levy, C., and Hartman, G. L. 2009. Effect of fungicide and timing of application on soybean rust severity and yield. Plant Dis. 93:243-248.

29. Posada-Buitrago, M. L., and Frederick, R. D. 2005. Expressed sequence tag analysis of the soybean rust pathogen Phakopsora pachyrhizi. Fungal Genet. Biol. 42:949-962.

30. Pryce-Jones, E., Carver, T., and Gurr, S. J. 1999. The roles of cellulase enzymes and mechanical force in host penetration by Erysiphe graminis $\mathrm{f}$. sp. hordei. Physiol. Mol. Plant Pathol. 55:175-182.

31. Stone, C. L., McMahon, M. B., Fortis, L. L., Nuñez, A., Smythers, G. W., Luster, D. G., and Frederick, R. D. 2012. Gene expression and proteomic analysis of the formation of Phakopsora pachyrhizi appressoria. BMC
Genomics 13:269-295.

32. Takano, Y., Kubo, Y., Shimizu, K., Mise, K., Okuno, T., and Furusawa, I. 1995. Structural analysis of $P K S 1$, a polyketide synthase gene involved in melanin biosynthesis in Colletotrichum lagenarium. Mol. Gen. Genet. 249:162-167.

33. Terhune, B. T., Bojko, R. J., and Hoch, H. C. 1993. Deformation of stomatal guard cell lips and microfabricated artificial topographies during appressorium formation by Uromyces. Exp. Mycol. 17:70-78.

34. Tremblay, A., Hosseini, P., Li, S., Alkharouf, N. W., and Mathews, B. 2013. Analysis of Phakopsora pachyrhizi transcript abundance in critical pathways at four time-points during infection of a susceptible soybean cultivar using deep sequencing. BMC Genomics 14:614. http://www. biomedcentral.com/1471-2164/14/614

35. Tremblay, A., Li, S., Scheffler, B., and Mathews, B. 2009. Laser capture microdissection and expressed sequence tag analysis of uredinia formed by Phakopsora pachyrhizi, the causal agent of Asian soybean rust. Physiol. Mol. Plant Pathol. 73:163-174.

36. Tsai, H.-F., Wheeler, M., Chang, Y., and Kwon-Chung, K. 1999. A developmentally regulated gene cluster involved in conidial pigment biosynthesis in Aspergillus fumigatus. J. Bacteriol. 181:6469-6477.

37. Vittal, R., Paul, C., Hill, C. B., and Hartman, G. L. 2014. Characterization and quantification of fungal colonization of Phakopsora pachyrhizi in soybean genotypes. Phytopathology 104:86-94. 